\title{
RESONANT DIAPHRAGM PRESSURE MEASUREMENT SYSTEM WITH ZnO ON Si EXCITATION*
}

J G SMITS, H A C TILMANS, $K$ HOEN, H MULDER, J VAN VUUREN and G BOOM

Department of Electrical Engineering, Twente University of Technology, Enschede (The Netherlands)

\begin{abstract}
The principle of measuring pressure by means of a resonant diaphragm has been studied An oscillator consisting of an integrated amplifier with a piezoelectrically driven diaphragm in its feedback loop has been built The oscillator frequency is accurately proportional to the square of the pressure in the range 60 to 130 Torr

The frequency range is 1324 to $1336 \mathrm{~Hz}$ (this range being limited by a spurious mode which could be suppressed by better processing) for a $25 \mathrm{~mm}$ diameter diaphragm made of a silicon wafer and with PZT ceramics as driver and receptor We have made an integrated version $\left(1 \times 1 \mathrm{~mm}^{2}\right)$ of a square resonant diaphragm pressure gauge by selective etching of $\left(\begin{array}{lll}1 & 0 & 0\end{array}\right)$ planes with ethylenediamine The piezoelectric driving material was sputtered zinc oxıde A driver was deposited midway between the bending point and the point of greatest curvature

A receptor was located at a symmetrical position to give an optimum transfer condition

The integrated current amplifier had a low impedance differential input stage, two gain cells and a high impedance output stage These electrical conditions ensured maximum elastic freedom of the diaphragm A digital circuit in $I^{2} \mathrm{~L}$ technology has been designed and made with elght-bit parallel read out of the frequency This crrcuit may be directly connected to a microprocessor The whole system contains the sensor chip, the analog amplifier chip and the digital chıp, all in compatible technology
\end{abstract}

\section{Introduction}

It has been shown that a pressure transducer can be made from a resonating diaphragm whose resonance frequency will change due to a static

* Based on a Paper presented at Solid-State Transducers 83, Delft, The Netherlands, May 31 - June 3, 1983 
deflection such as that caused by pressure or force The effect of pressure, for example, is to increase the potential energy and hence produce a higher resonance frequency [1] The dependence of the resonance frequency on the pressure is given by

$f=f_{0}\left(1+\alpha p^{2}\right)^{1 / 2}$, or $\frac{f^{2}-f_{0}^{2}}{f_{0}^{2}}=\alpha p^{2}$ (parabolic dependence)

where $f_{0}$ is the resonance frequency at zero pressure, $p$ is the pressure and $\alpha$ is the sensitivity

The schematic diagram of the system contans four sections (see Fig 1)

(1) The diaphragm with a piezoelectric exciter and receptor

(2) An amplifier that forms an oscillator if it has the diaphragm in its feedback loop word

(3) A creuit that converts the frequency of this oscillator into a data

(4) A reference oscillator

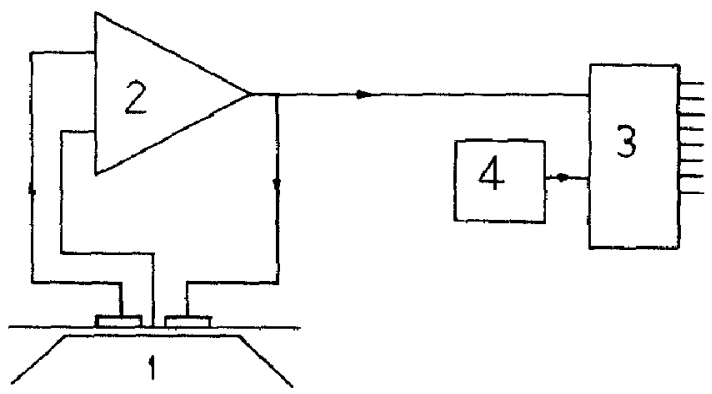

Fig 1 Schematic diagram of the pressure measurement system

The principle of operation is as follows If we drive the prezoelectric exciter with an electrical signal, it expands and contracts in the rhythm of the driving signal, if it is free Here, as it is attached to the diaphragm, the exciter exerts forces and moments, which bend and elongate or contract the diaphragm A second piezoelectric transducer will pick up this motion and give a signal proportional to its elongation or contraction If driven at the fundamental resonance frequency of the diaphragm, the output signal becomes very large, so that we may treat the system as a filter

The maximum in the transfer function of the filter will shift to higher frequencies if pressure is applied to the diaphragm, due to its stiffening, the maximum itself will also increase because it is a non-linear system

By placing this filter in the feedback loop of an amplifier, we can make an oscillator with a frequency suitable for digital processing A full-size wafer diaphragm (see Fig 2) and amplifier have been used to perform experiments on the frequency change of the oscillator versus the pressure The pressure on the diaphragm was determmed with an accuracy of approximately $1 \mathrm{~mm}$ of $\mathrm{H}_{2} \mathrm{O}$, while the frequency was measured with a precision of $01 \mathrm{~Hz}$ The frequency initially decreases with increasing pressure (Fig 3 ) because the 


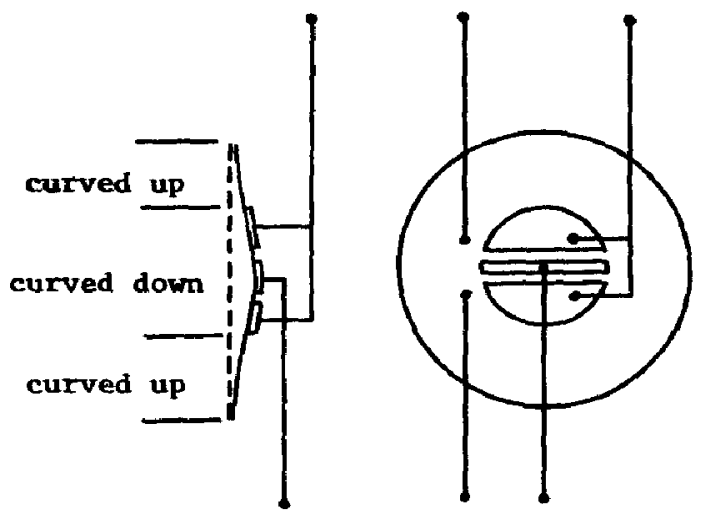

Fig 2 A full sized silicon wafer with two exciters and one receptor

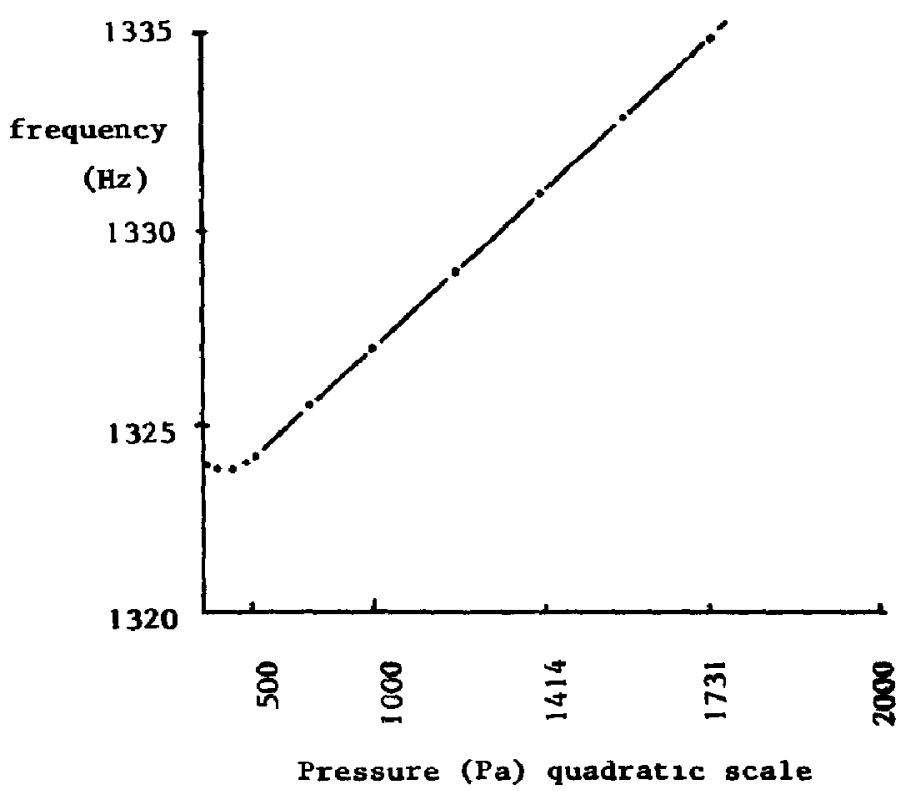

Fig 3 Oscillation frequency versus square of pressure for a prototype 2 " wafer as resonant diaphragm

adhesive with which the transducers were attached to the diaphragm is a two-component thermocuring epoxy mixture which cures at $100{ }^{\circ} \mathrm{C}$ After cooling to room temperature, there will be a static deflection due to the difference in thermal expansion coefficients of PZT $\left(\approx 6 \mathrm{ppm} /{ }^{\circ} \mathrm{C}\right)$ and silicon $\left(\approx 3 \mathrm{ppm} /{ }^{\circ} \mathrm{C}\right)$ The pressure was appled to the convex side of the diaphragm, so this thermal deflection had to be reduced by the pressure before the diaphragm could achieve its flat form The thermal deflection could be represented here as an internal pressure which had to be overcome before the theoretical parabolic dependence could emerge

The parabolic dependence starts at approximately $30 \mathrm{~cm} \mathrm{H}_{2} \mathrm{O}$ and continues up to $170 \mathrm{~cm} \mathrm{H}_{2} \mathrm{O}$ At this point the frequency jumped to a higher value due to a spurious mode in the resonance loop (not shown in the 
Figure) This spurious mode was due to a slight non-symmetrical positioning of the driving transducers with respect to the sensing transducer, and could have been avolded by more careful processing of the draphragm The accuracy of this diaphragm as a sensor is better than the over-all accuracy of the system, which was limited to $1 \%$ because of the counter resolution Estimates of the sensor accuracy from the scattering of the measuring points around a least-square-fitted straight line indicate that this is one order of magnitude better

The components of the system will now be discussed briefly

\section{The diaphragm}

This has now been made in three different versions Diaphragms were furst made out of a full $2^{\prime \prime}$ silicon wafer and a thin brass plate to study the pressure sensitivity of the resonance frequency A sensor of chip-size dimensions, which is presented here, was then made To investigate the transfer function of these kinds of systems, theoretical and experimental studies of beams which are excited and sensed by piezoelectric transducers [3] have been performed A thick $5 \mu \mathrm{m}$ diaphragm has been made by preferential etching of a (100) oriented silicon wafer (Fig 4) The wafers were covered with a thermal oxide approximately $1 \mu \mathrm{m}$ thick, which formed the etching mask for etching of the holes in the unpolished back side of the wafer The etchant was ethylenediamine pyrocatechol (EDP) The etching procedure was a three-step process first, a rough etch at $90^{\circ} \mathrm{C}$ until the majority of the silicon had been removed, secondly a fine etching step at $30^{\circ} \mathrm{C}$ to determine the etching rate at room temperature and finally the last microns were removed The diaphragm had been reinforced by a $05 \mu \mathrm{m}$ thick layer of boron glass, deposited in a CVD process

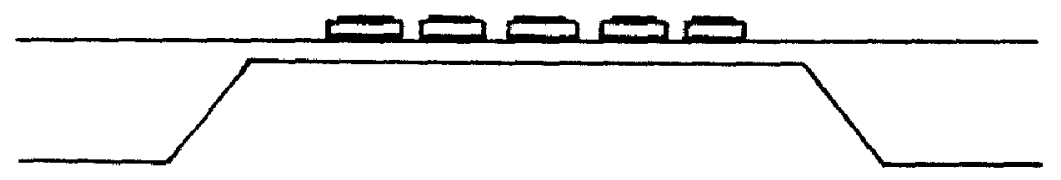

Fig 4 Schematic cross section of sensor

The transducers were arranged in three concentric squares (Fig 5) which had to be opened to allow the leads of the electrodes to reach the two inner transducers We have chosen this configuration to make it possible to use, $e g$, the outer and centre transducers as exciters and the middle transducer as receptor, or another combination to find an optimum excitation condition Another concept which we want to investigate is the possibility of counteracting the pressure action by means of the moments and forces that can be exerted by the piezoelectric ZnO at a static electric field In this way it should be possible optimize the pressure sensitivity 


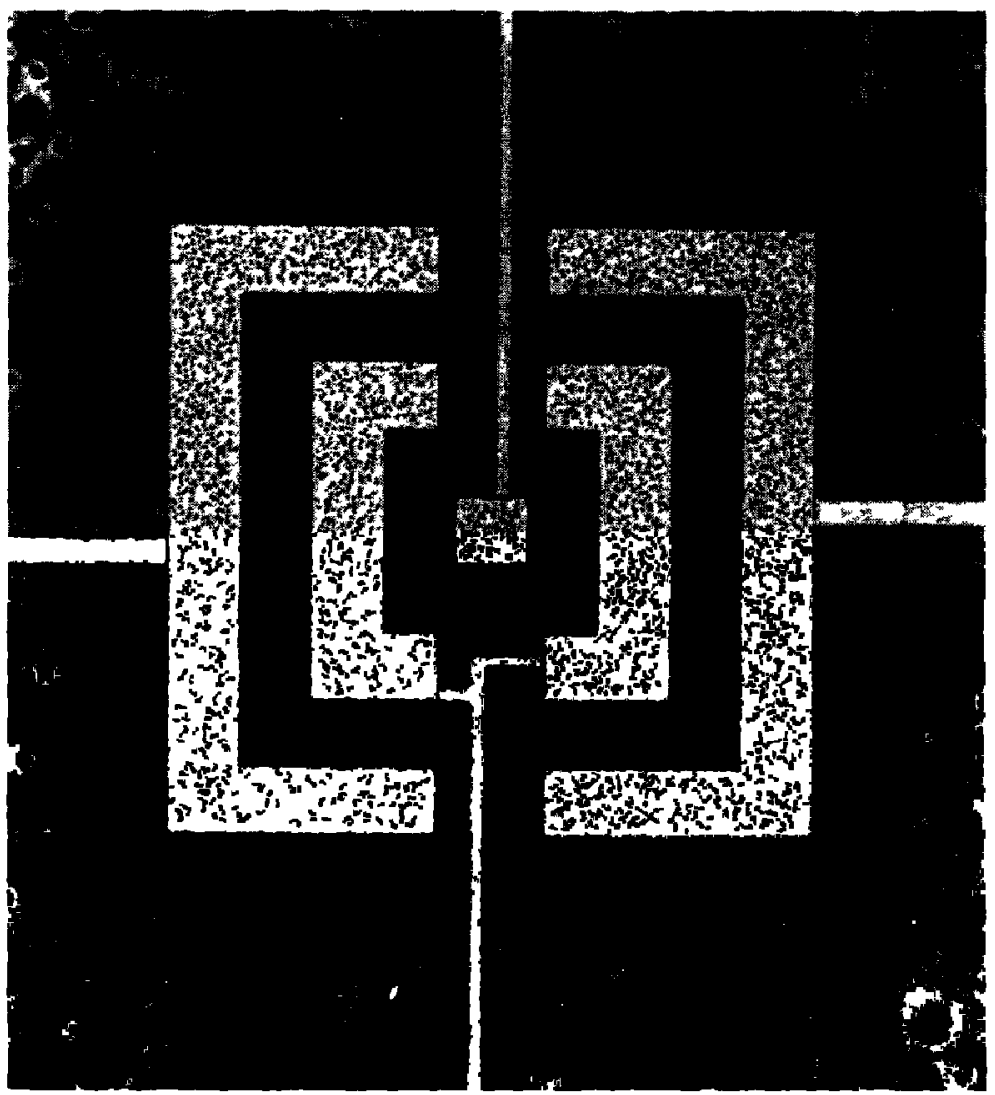

Fig 5 The sensor with $\mathrm{ZnO}$ excitors and receptors

\section{The amplifier}

A current amplifier and a transimpedance amplifier have been designed for use with the micro diaphragms (see Fig 6) [3] The latter has been tested with the beam as well as with the full size diaphragms The current input and output of the current amplifier were chosen in order to satisfy the electrical boundary conditions of the diaphragm driving it with a force source instead of with a deformation source at its input, and sensing it with a maximally weak sensor The terms force source and deformation source are the mechan1cal analogs of voltage and current sources a force source will maintain its force irrespective of the displacement of the subject on which it acts and vice versa for a deformation source

\section{The counter}

This is an eight bit frequency counter, which determmes the frequency difference between the measured frequency input and a reference frequency input (see Fig 7) 


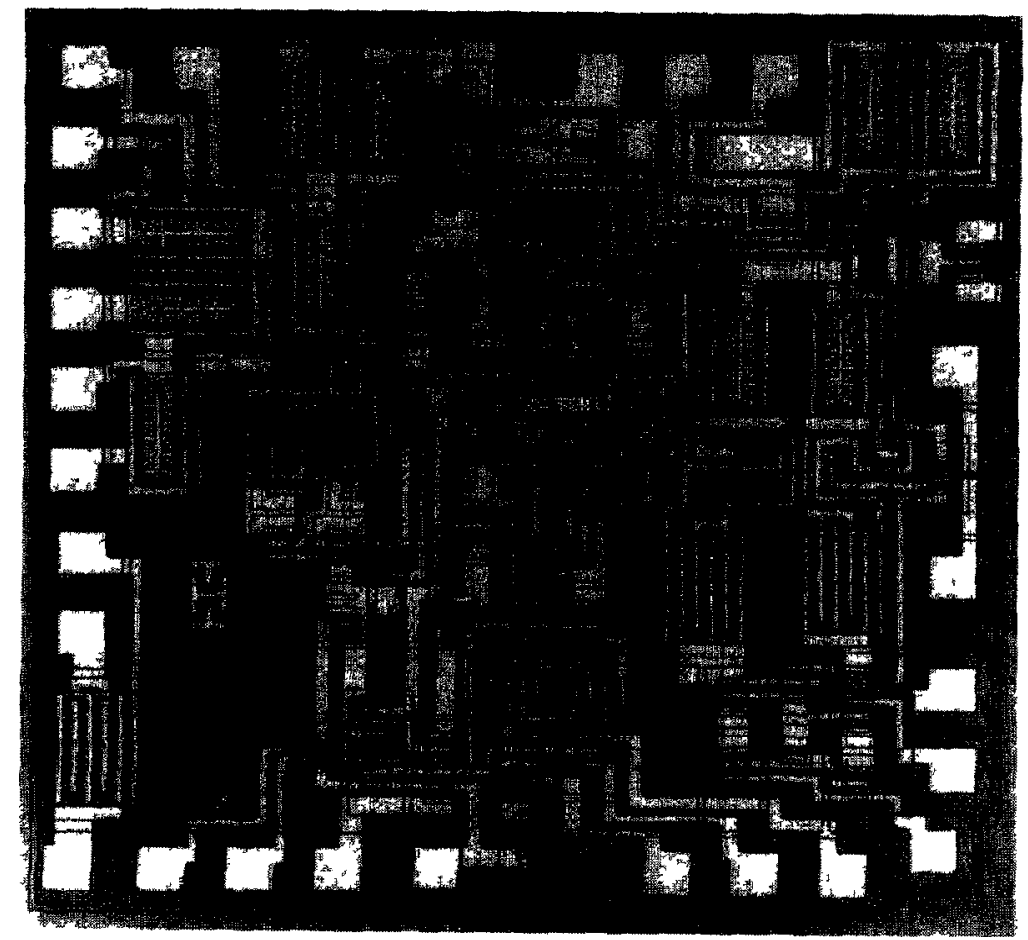

Fig 6 Current amplifier in bipolar technology

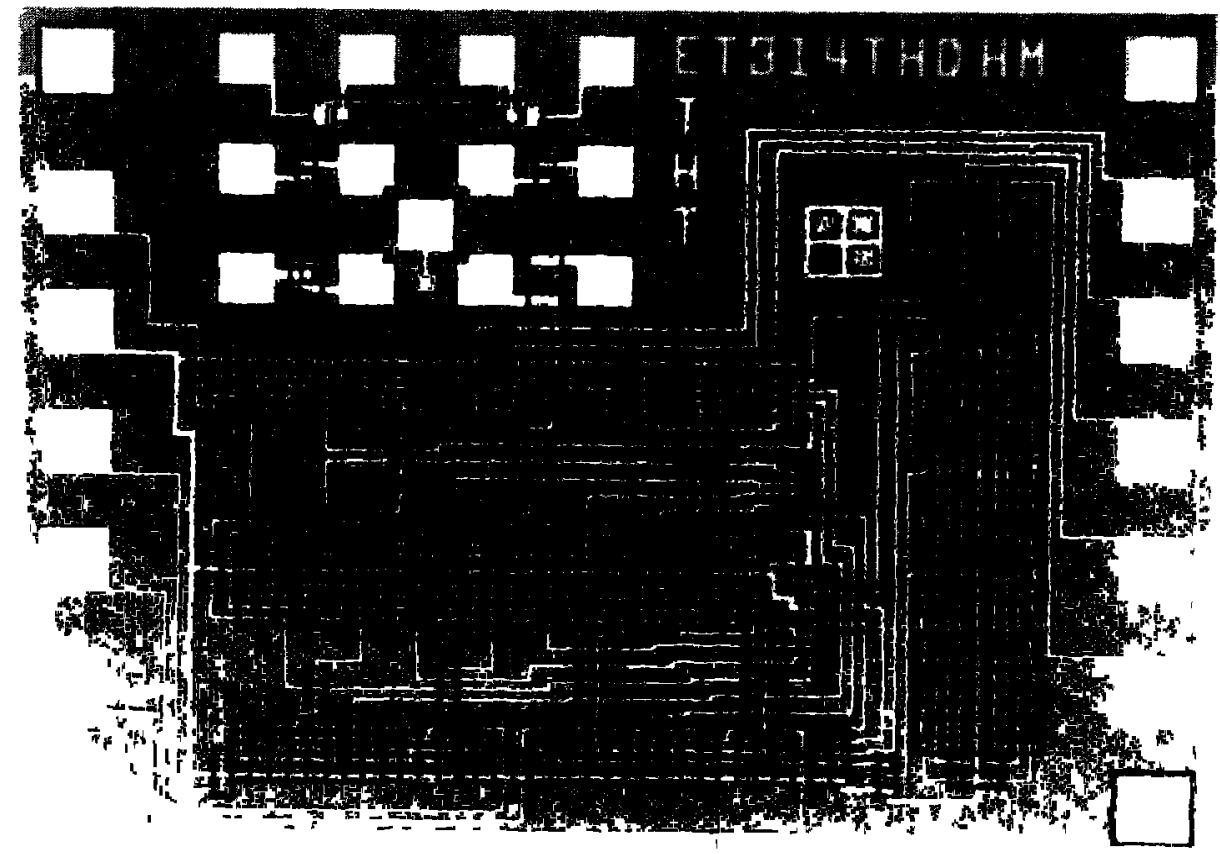

Fig $7 \mathrm{I}^{2} \mathrm{~L}$ countercircuit 
It was designed and realized in $\mathrm{I}^{2} \mathrm{~L}$ technology [2] It contains $105 \mathrm{I}^{2} \mathrm{~L}$ transistors and has a current consumption of $3 \mathrm{~mA}(20 \mu \mathrm{A} /$ transistor $)$ It can handle frequencies up to several hundred kilohertz It has an eight-bit parallel output and it is directly microprocessor compatible

\section{The reference osculator}

The reference input of the counter is an identical diaphragm with amplifier combination on which there are no pressure changes In this way the temperature variations in the resonance frequency can be compensated

\section{References}

1 S Timoshenko, Vibration Problems in Engineering, Van Nostrand, Princeton, N J, 1955

$2 \mathrm{H}$ Mulder, Resonant diaphragm pressure sensor, Technical Report 12223573 , T H Twente, 1983

3 M Vertregt, Design and realization of a current amplifier for a pressure sensor, Technical Report $12223537, \mathrm{~T}$ H Twente, 1982 